\title{
Clinical Trials Corner: Nephrectomy in the New Year
}

Received 11 January 2022

Accepted 17 January 2022

Pre-press 14 December 2020

Published 15 March 2022

Dear Readers,

The Clinical Trials Corner of Kidney Cancer highlights planned or ongoing high-impact studies in renal cell carcinoma (RCC). In this issue, we highlight the NORDIC-SUN trial evaluating the role of deferring cytoreductive nephrectomy and will contrast this trial to a previously mentioned SWOG PROBE trial.

In the future, if you feel that you would like to draw attention to a specific trial, please feel free to email us at mbparikh@ucdavis.edu orkca@iospress.com.

Sincerely,

Mamta Parikh, MD, MS

Associate Editor, Kidney Cancer

Assistant Professor, University of California Davis School of Medicine

Department of Internal Medicine

Division of Hematology Oncology

Sacramento, California

Multicenter Randomized Phase III Trial of Deferred Cytoreductive Nephrectomy in Synchronous Metastatic Renal Cell Carcinoma Receiving Checkpoint Inhibitors: a DaRenCa and NoRenCa Trial Evaluating the Impact of Surgery or No Surgery. The NORDIC-SUN-Trial.

Status: Recruiting

Clinicaltrials.gov identifier: NCT03977571

Sponsor: Frede Donskov, Aarhus University Hospital

Enrollment: 400

Rationale: The benefit of cytoreductive nephrectomy $(\mathrm{CN})$ had previously been established among fit patients with metastatic renal cell carcinoma during the era of interferon alfa treatment. Subsequently, however, systemic therapy for mRCC evolved significantly. During the era of single agent anti-angiogenesis therapy as first-line treatment, the CARMENA trial supported the deferral of $\mathrm{CN}$ in patients receiving sunitinib who had intermediate or poor-risk disease. Yet at the same time, the current standard of care treatment for mRCC has evolved to include immune checkpoint inhibitors in first-line treatment. As such, the role of cytoreductive nephrectomy remains of question, prompting both the SWOG PROBE trial, and this trial, NORDIC-SUN. 
Study Design: This Phase III multi-site, randomized trial enrolls patients with histologically proven clear cell or non-clear cell synchronous metastatic RCC who have not previously received systemic therapy. Patients are eligible if considered to be appropriate for treatment with nivolumab plus ipilimumab according to the recommendations by the European Medicines Agency and national health authorities of any participating countries. Patients who have autoimmune disease requiring systemic corticosteroids, and patients with HIV, Hep B or Hep C are excluded from participation in this trial. Patients enrolled to study will be randomized to either be treated with nivolumab plus ipilimumab (every 3 weeks for 4 doses) then undergo cytoreductive nephrectomy followed by maintenance nivolumab every 4 weeks or be treated with nivolumab plus ipilimumab (every 3 weeks for 4 doses) followed by nivolumab every 4 weeks. All patients participating will have archival tissue collected as well as blood and stool specimens for translational biomarker research testing.

Endpoints: The primary endpoint of this trial is overall survival (OS). The secondary outcomes include progression-free survival (PFS), objective response rate (ORR), and time to subsequent systemic therapy. Biomarker analyses will include evaluation of tumor infiltrating lymphocytes (TILs) at baseline and after surgery, immune subsets in blood measured by flow cytometry, genetic profile of circulating tumor DNA, and profile of gut microbiome.

Comments: This cooperative group sponsored multi-center Phase II trial evaluates RCC patients with bone metastases, a population with poor outcomes both in terms of morbidity and mortality. Importantly, while Radium-223 dichloride has been studied alone in patients with prostate cancer and in combination with antiangiogenesis agents in patients with $\mathrm{mRCC}$, this study will better evaluate whether there is a benefit to Radium-223 added to treatment. Prior studies did not have a control arm, and this study has a cabozantinibalone comparator arm. With correlative analysis of markers of bone turnover in this study, there is potential to further understanding of the mechanism of the effect of both Radium-223 dichloride and cabozantinib on osseous metastases. Importantly, the study chairs have recently updated the eligibility criteria as outlined in the Study Design, allowing now for patients who only have one untreated bony lesion and for patients who are asymptomatic from osseous metastases. The trial eligibility requirements have also been adjusted to allow for a hemoglobin threshold of $>9$. These changes should encourage readers to consider this important trial when evaluating a patient with progressive osseous metastases. The challenge in this trial, of course, is that data from trials like METEOR have led to an increased use of cabozantinib early in the treatment of mRCC patients with bony metastases, and may lead some to even use first-line nivolumab plus cabozantinib on the basis of the CheckMate-9ER study. However, it is important to note that these studies stratified for osseous metastases, in contrast to the RADICAL study, which specifically studies mRCC patients with a preponderance of bony metastases.

\section{CONFLICT OF INTEREST}

Mamta Parikh

Consultant: Janssen, Seagen, AstraZeneca, Oncocyte 